\title{
Using Robot Manipulation to Assist Navigation by People Who Are Blind or Low Vision
}

\author{
Xiang Zhi Tan \\ Robotics Institute \\ Carnegie Mellon University \\ Pittsburgh, USA \\ zhi.tan@ri.cmu.edu
}

\author{
Aaron Steinfeld \\ Robotics Institute \\ Carnegie Mellon University \\ Pittsburgh, USA \\ steinfeld@cmu.edu
}

\begin{abstract}
This work explores the capability of bi-directional manipulation during assistance to blind or low vision users. We describe a haptic approach that utilizes the manipulator arm to support interaction and provide navigational information supplemented with landmarks and spatial cues to users.

\section{Keywords}

Assistive Robots; Haptic Interface; Indoor Navigation

\section{INTRODUCTION}

Previous work done in the realm of human-robots interaction with people who are blind or low vision(B/LV) has generally focused on mobile robots [2] or bootstrapping existing robotic technology onto other devices $[3,4]$. Compared with other technological solutions, the possible design space for robots with manipulation capabilities has been largely left unexplored. Our research aims to examine this space by creating services that use new technology while grounded by continuous user feedback from relevant stakeholders. We utilize a model where the robot provides services to occupants and visitors but is owned and managed by the building owners. This simplifies cost and implementation challenges. This type of robot is commonly categorized as a building service robot [1]. We envision a manipulator robot as a kiosk desk robot that remains stationary provides information and services to users. However, working with the B/LV population presents new challenges that are overlooked in existing systems. In this paper, we describe completed and current work on alternative interaction methods and services that our robot can provide for these users. We choose to use the Baxter platform due to it's compliant nature, lack of pinch points, and design for collaborative work.
\end{abstract}

\section{ALTERNATIVE HAPTIC INTERFACE}

One challenge faced in designing services between users and robots is the limitation in methods of interaction. Social

\footnotetext{
Permission to make digital or hard copies of part or all of this work for personal or classroom use is granted without fee provided that copies are not made or distributed for profit or commercial advantage and that copies bear this notice and the full citation on the first page. Copyrights for third-party components of this work must be honored. For all other uses, contact the owner/author(s).

HRI'17 Companion March 06-09, 2017, Vienna, Austria

(C) 2017 Copyright held by the owner/author(s).

ACM ISBN 978-1-4503-4885-0/17/03.

DOI: http://dx.doi.org/10.1145/3029798.3034808
}

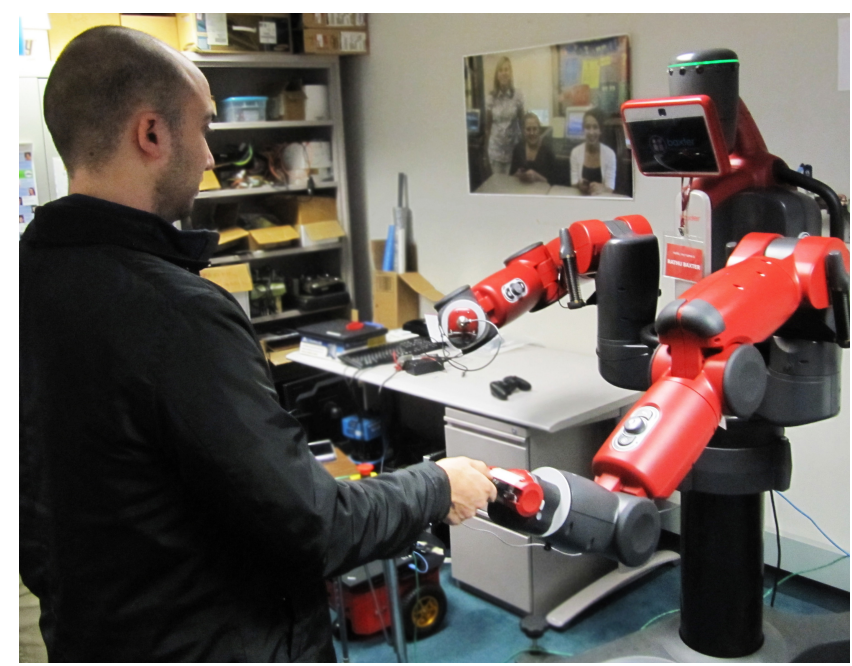

Figure 1: Potential user interacting with our robot

interaction between users and robots are often done through visual or audio channels. However, visual based interaction are unsuitable for our target user group, whereas relying solely on speech recognition might be infeasible due to noisy environment. Our recently completed work explored the possibility of communication through an haptic interface that relies on existing hardware [5].

We explored a interaction technique that uses the robot's arm as an input device that users can move to communicate their intent. The technique is analogous to interacting with a manipuladom or a joystick. We believe the strength of the system lies in its use of an existing robot component (reduced cost), ability to retain contact with the arm during interaction (reduced confusion), and the wide range of tasks which can be accomplished with an arm (task flexibility).

We created a two part system to facilitate this type of interaction. The first component is a feedback mechanism. It maintains the robot's arm in place and mimics the feedback of a joystick by applying resistance as the user deviates from the starting position using a PD controller. This feedback is designed to guide the user's movement. The second component is a gesture classification system. It takes in the movement at each time step and classifies them into predefined gestures that are used as input for the desired application. In our work, we explored two classification methods, (1) decision list and (2) linear machine learning techniques (SVM and Logistic Regression). Both approaches perform 
well with an accuracy of about $90 \%$ in our experiments. Performance increased further when we added audio feedback after gestures are recognized. While both methods performed well, we believe a machine learning approach might be more suitable for a wider audience because of the capability to learn the decision boundaries from a non-programmer during installation or directly from the users [5].

We performed user study and found there is no significant difference in time taken to complete a task when compared to a keypad input. The participants were asked to use the interface to select an option from a series in a list using both input methods. Our haptic interface had a mean time of $61.9 \mathrm{~s}(\mathrm{SD}=23.7 \mathrm{~s})$, while the keypad input had a mean of $65.8 \mathrm{~s}(\mathrm{SD}=48.4 \mathrm{~s})$. We also found no significant difference between interfaces with our user subjective metrics.

\section{PROVIDING DIRECTIONAL INFORMATION TO USERS}

Like an information desk clerk, we have designed the robot to provide verbal directions for routes to a destination. The user first selects a destination using the interaction method described above. Our robot then obtains a route from a path-planning server and presents this information to the user as a list of verbal instructions. An example of a single instruction would be "turn to 2 o'clock and walk about 100 feet forward". Beyond verbal directions, we are also exploring techniques to provide more helpful information, especially for $\mathrm{B} / \mathrm{LV}$ users. We are working on two additional components - (1) physical spatial directions and (2) on-demand rich landmark information.

Our approach provides an alternative to the hand gestures often used when directing sighted people (e.g., "Go that way"). Instead, we provide physical spacial directions inspired by the technique used by Orientation and Mobility experts where they draw a path on a blind person's palm. Instead of drawing routes directly on the palm, we ask our users to hold onto the end effector of our robot while the robot makes redundant motions in a small space between the robot and user. For example, the instruction "turn to 2 o'clock and walk 100 feet forward" is decomposed into two parts. First, the robot rotates it's end effector to the 2 o'clock direction. Next, it moves along that direction to simulate the travel of 100 feet. We scaled the relationship between the movement and the actual distance to give a sense of distance without overextending the workspace. An example of this movement is shown in Figure 2.

When describing a route to a sighted person, people will often include visual landmarks like signs and colors to assist with localization and memory. Landmarks are also useful for people who are B/LV, but they need different kinds of information and often have specific preferences. Specifically, audio or haptic landmarks like sound of machines and change in carpets are useful. At any point while the robot is providing verbal and gesture instructions, the user can use its arm as a haptic input interface by simply moving in a specific direction. The robot will pause the current instruction and describe possible landmarks for that point in the path. Our goal is to describe landmarks that will be most helpful for that particular user based on their preference and capabilities. Other gestures are used to repeat navigation instructions. Unlike a human, robots are willing to repeat route instructions for as long as the user desires.

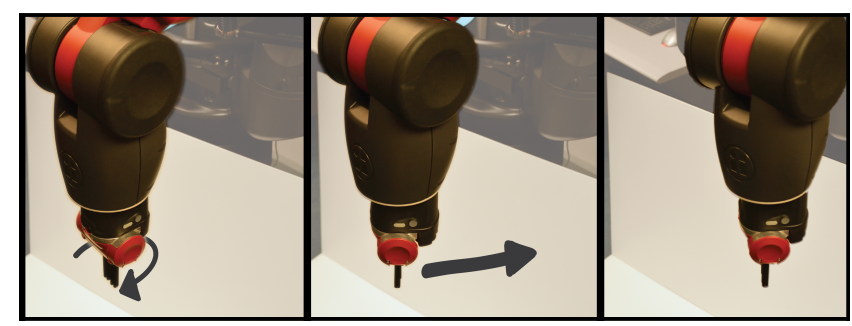

Figure 2: Examples of end effector movement when providing spatial directions. From the left, the end effector first rotates to face the 2 o'clock direction. It then moves in that direction to convey the estimate distance to the next instruction point.

We use an iterative design approach in our creation of the system, where B/LV users are invited to test our system during the implementation phase. Some initial feedback we have received includes integrating useful landmarks, like hallways, directly into the instructions and providing more opportunities for users to customize the interaction based on their mental model and preferences. We are currently improving the system based on these feedback.

\section{CONCLUSION}

As we continue develop our system, we envision our system to not only provide navigational information for users to get from one place to another but also providing other types of services. Possible future services include expanding upon the robot's vision capabilities, like sorting transit tickets. We believe no one should be left behind as robot technology advances and is deployed in the wild.

\section{ACKNOWLEDGMENT}

This work was funded by a grant (National Robotics Initiative, IIS-1317989) from the National Science Foundation. The authors also thank the participants in the user studies.

\section{REFERENCES}

[1] S. Azenkot, C. Feng, and M. Cakmak. Enabling building service robots to guide blind people a participatory design approach. In Proc. HRI, pages 3-10, 2016.

[2] V. Kulyukin, C. Gharpure, J. Nicholson, and G. Osborne. Robot-assisted wayfinding for the visually impaired in structured indoor environments. Autonomous Robots, 21(1):29-41, 2006.

[3] S. Maidenbaum, S. Hanassy, S. Abboud, G. Buchs, D. R. Chebat, S. Levy-Tzedek, and A. Amedi. The 'EyeCane', a new electronic travel aid for the blind: Technology, behavior \& swift learning. Restorative Neurology and Neuroscience, 32(6):813-824, 2014.

[4] B.-C. Min, S. Saxena, A. Steinfeld, and M. B. Dias. Incorporating information from trusted sources to enhance urban navigation for blind travelers. In Proc. ICRA, pages 4511-4518, 2015.

[5] X. Z. Tan, A. Steinfeld, and M. B. Dias. Communication through movement: An alternative method of interaction for HRI. In R:SS 2016 workshop on Socially and Physically Assistive Robotics for Humanity, 2016. 